\title{
THE HOCHSCHILD HOMOLOGY OF COMPLETE INTERSECTIONS
}

\author{
BY
}

\section{KLAUS WOLFFHARDT}

ABSTRACT. Let $\widetilde{R}$ be the algebra of all convergent (or of all strictly convergent) power series in $N$ variables over a commutative field $K$ of characteristic 0 with a valuation, e.g. $\widetilde{R}=K\left[X_{1}, \cdots, X_{N}\right]$. With each $K$-algebra $R \cong \widetilde{R} / a$ we associate a bigraded $R$-algebra $E$. By the powers of $a$ a filtration of the Poincaré complex of $\widetilde{R}$ is induced, and $E$ is the first term of the corresponding spectral sequence. If $a$ is generated by a prime sequence in $\widetilde{R}, R$ is called a complete intersection, and $E$-with an appropriate simple grading-is isomorphic to the Hochschild homology of $R$. The result is applied to hypersurfaces.

1. Introduction. Let $K$ be a fixed field of characteristic 0 with a multiplicative valuation $x \mapsto|x| \in \mathbf{R}$. A formal power series $\Sigma c_{k_{1}} \cdots k_{N} X_{1}^{k_{1}} \cdots X_{N}^{k}$ is called convergent (strictly convergent) if $\Sigma\left|c_{k_{1}} \ldots k_{N} x_{1}^{k} \ldots x_{N}^{k}\right|<\infty$ for all $x_{1}, \cdots, x_{N}$ with $\left|x_{i}\right| \leq \epsilon$ for some $\epsilon>0$ (resp. for $\epsilon=1$ ). Let $K\left\{X_{1}, \cdots, X_{N}\right\}$ denote the $K$-algebra of all power series in $X_{1}, \cdots, X_{N}$ that are convergent ("analytic case") or strictly convergent ("affinoid case"-for nonarchimedean valuations only). In this paper we will not need different notations for the two cases. Observe that for the trivial valuation of $K$ the analytic case reduces to the "formal case" (the algebra of all formal power series), and the affinoid case reduces to the "affine case" (the polynomial algebra). The category of all nonzero $K$-algebras $K\left\{X_{1}, \cdots, X_{N}\right\} /$ a with morphisms of $K$-algebras which map the identity element onto the identity element is called the category of analytic (resp. affinoid) $K$-algebras. In the following we shall always speak about analytic $K$-algebras-but all proofs and theorems are valid in the affinoid case as well.

In the category of analytic $K$-algebras there exist finite direct sums: If $R=$ $K\left\{X_{1}, \cdots, X_{N}\right\} / a$ and $R^{\prime}=K\left\{X_{1}^{\prime}, \cdots, X_{N^{\prime}}^{\prime}\right\} / b$ let $c$ be the ideal generated by $a+\mathfrak{b}$ in the $K$-algebra $K\left\{X_{1}, \cdots, X_{N}, X_{1}^{\prime}, \cdots, X_{N^{\prime}}^{\prime}\right\}$ of convergent power series in indeterminates $X_{1}, \cdots, X_{N^{\prime}} X_{1}^{\prime}, \cdots, X_{N^{\prime}}^{\prime}$. Then $R \Perp R^{\prime}:=$ $K\left\{X_{1}, \cdots, X_{N}, X_{1}^{\prime}, \cdots, X_{N}^{\prime}\right\} / C$ is a direct sum of $R$ and $R^{\prime}$. There are canonical morphisms from $K\left\{X_{1}, \cdots, X_{N}\right\}$ and from $K\left\{X_{1}^{\prime}, \cdots, X_{N^{\prime}}^{\prime}\right\}$ into $K\left\{X_{1}, \cdots, X_{N}, X_{1}^{\prime}, \cdots, X_{N}^{\prime}\right\}$; these induce the canonical morphisms from $R$ and $R^{\prime}$ into $R \Perp R^{\prime} . R \Perp R^{\prime}$ is called the analytic tensor product of $R$ and $R^{\prime}$, and

Received by the editors June 1,1971 .

AMS 1969 subject classifications. Primary 1390, 1393, 3210, 3280.

Key words and phrases. Hochschild homology, analytic algebra, affinoid algebra, affine algebra, formal differentials, complete intersection, hypersurface. 
$R \otimes R^{\prime}$ may be considered a $K$-subalgebra of $R \Perp R^{\prime}$.

For an analytic $K$-algebra $R$ we call $S:=R \Perp R$ the enveloping algebra of $R$. There is a unique morphism $\pi: S \rightarrow R$, such that for both canonical maps $R \rightarrow R \Perp R$ the composition $R \rightarrow S \rightarrow \pi R$ is the identity. $\pi$ is called the augmentation of $S$. In virtue of $\pi$ we consider $R$ (and every $R$-module) as an $S$-module. According to [1] the Hochschild homology of $R$ with coefficients in $R$ is the graded $K$-module $\operatorname{Tor}^{S}(R, R)=: \Omega(R)$; it is an anticommutative graded $R$-algebra [3]. There is a mapping $d: R \rightarrow \Omega^{1}(R)$ which is universal as a $K$-derivation from $R$ to a finitely generated $R$-module (see [2] for instance). Hochschild, Kostant and Rosenberg [3] showed that $\Omega(R)$ may be considered as the algebra of formal differentials of $R$ over $K$. From a direct calculation it follows that $\Omega(R)=\bigwedge_{R} \Omega^{1}(R)$ if $R=K\left\{X_{1}, \cdots, X_{N}\right\}$. In [3] this equation is proved for $R$ a regular affine domain. We showed in [8] that in the affine case the natural morphism $\bigwedge_{R} \Omega^{1}(R) \rightarrow \Omega(R)$ is injective.

In this paper we will, for each analytic $K$-algebra $R$, define a differential bigraded algebra $E(R)$, which can be calculated easily from the knowledge of an isomorphism $R \cong \tilde{R} / a, \widetilde{R}=K\left\{X_{1}, \cdots, X_{N}\right\}$ : The powers of the ideal a induce a filtration of the Poincaré complex $\Omega(\tilde{R})$ of the $K$-algebra $\tilde{R}$, and $E(R)$ is the first term of the spectral sequence defined by this filtration. In particular, $\bigwedge_{R} \Omega^{1}(R)$ is a subalgebra of $E(R)$. We will prove that $E(R)$ with an appropriate simple grading is isomorphic to the graded algebra $\Omega(R)$ when $R$ is a complete intersection, i.e. when $a$ is generated by a prime sequence.

Some open questions: Is $E(R)$ a functor, and - if yes - are $E(R)$ and $\Omega(R)$ naturally isomorphic? Are $E(R)$ and $\Omega(R)$ isomorphic as differential graded algebras? (In the affine case a differentiation in $\Omega(R)$ has been defined explicitly by Rinehart [5].) What connection is there between $E(R)$ and $\Omega(R)$, when $R$ is not a complete intersection?

2. Definition of $E(R)$. Let $R$ be a given analytic $K$-algebra. There is a surjective morphism of $K$-algebras $\phi: \widetilde{\widetilde{R}} \rightarrow R$ with $\widetilde{R}=K\left\{X_{1}, \cdots, X_{N}\right\}$. Let $a:=$ ker $\phi$. Let $\tilde{\Omega}:=\Omega(\widetilde{R})=\bigwedge_{\widetilde{R}} \widetilde{\Omega}^{1}$ with the usual differential $\tilde{d}$, which is characterized as the derivation of degree +1 of the graded $K$-algebra $\widetilde{\Omega}$, such that $\tilde{d}^{0}$ is the universal derivation of $\widetilde{R}$ mentioned above.

For all $n, p \in \mathbf{Z}$ we have $\widetilde{d}\left(a^{p-n} \widetilde{\Omega}^{n}\right) \subset a^{p-(n+1} \tilde{\Omega}^{n+1}$ (let $a^{-q}:=\widetilde{R}$ for $q \in \mathbf{N})$. Hence, by $F^{p} \widetilde{\Omega}^{n}:=a^{p-n} \widetilde{\Omega}^{n}$, a descending filtration of the complex $\widetilde{\Omega}$ is defined. This filtration defines (cf. [1]) a spectral sequence of $K$-modules. We are interested only in the first term of this spectral sequence. It consists of the vector spaces

$$
E^{p, q}:=H^{p+q}\left(F^{p} \tilde{\Omega} / F^{p+1} \tilde{\Omega}\right)
$$


Trivially, $E^{p, q}=0$ if $p+q<0$ or $p+q>N$ or $q>0$.

We have

$$
\begin{aligned}
& F^{p} \tilde{\Omega}^{n} \wedge F^{p^{\prime}}{\widetilde{\Omega^{n}}}^{n^{\prime}}=a^{p-n}{\widetilde{\Omega^{n}}} \wedge a^{p^{\prime}-n^{\prime}}{\widetilde{\Omega^{n}}}^{\prime} \\
& C a^{p+p^{\prime}-\left(n+n^{\prime}\right)} \tilde{\Omega}^{n+n^{\prime}}=F^{p+p^{\prime}} \widetilde{\Omega}^{n+n^{\prime}},
\end{aligned}
$$

i.e. $F$ is a filtration of the $\tilde{R}$-algebra $\tilde{\Omega}$, and the $F^{p} \tilde{\Omega} / F^{p+1} \tilde{\Omega}$ form the associated graded $\tilde{R}$-algebra $G \tilde{\Omega}$. But $G \widetilde{\Omega}$ is an $R$-algebra because it contains $F^{0} \widetilde{\Omega}^{0} / F^{1} \widetilde{\Omega}^{0}$ $\cong R$ as an $\widetilde{R}$-subalgebra. $\tilde{d}$ induces a derivation on $G \tilde{\Omega}$, for which

$$
R \cong F^{0} \widetilde{\Omega}^{0} / F^{1} \widetilde{\Omega}^{0} \rightarrow F^{0} \widetilde{\Omega}^{1} / F^{1} \widetilde{\Omega}^{1}
$$

is the zero mapping because $F^{0} \widetilde{\Omega}^{1}=F^{1} \widetilde{\Omega}^{1}$. Hence this derivation is $R$-linear, and $H(G \widetilde{\Omega})=\left(E^{p, q}\right)_{p, q \in Z}$ is an $R$-algebra with $E^{0,0}=R$ and, more generally,

$$
\left(E^{p, 0}\right)_{p \in Z}=\bigwedge_{R} \Omega^{1}(R) .
$$

If $x \in E^{p, q}$ and $x^{\prime} \in E^{p^{\prime}, q^{\prime}}$ are represented by $y \in F^{p} \widetilde{\Omega}^{n}$ and $y^{\prime} \in F^{p^{\prime}} \widetilde{\Omega}^{n^{\prime}}$ respectively $\left(n=p+q, n^{\prime}=p^{\prime}+q^{\prime}\right)$, then $x \wedge x^{\prime}$ is represented by $y \wedge y^{\prime} \epsilon$ $F^{p+p^{\prime}} \widetilde{\Omega}^{n+n^{\prime}}$ and $x^{\prime} \wedge x$ by $y^{\prime} \wedge y=(-1)^{n n^{\prime}} y \wedge y^{\prime}$. Hence the multiplication

$$
E^{p, q} \times E^{p^{\prime}, q^{\prime}} \rightarrow E^{p+p^{\prime}, q+q^{\prime}}
$$

is anticommutative with respect to the total degree $n$ :.

$$
x \wedge x^{\prime}=(-1)^{n n^{\prime}} x^{\prime} \wedge x
$$

To summarize these results we use

Definition. An anticommutative bigraded $R$-algebra is a bigraded $R$-module $\left(E^{p, q}\right)_{p, q \in Z}$ together with a family of $R$-bilinear mappings

$$
E^{p, q} \times E^{p^{\prime}, q^{\prime}} \rightarrow E^{p+p^{\prime}, q+q^{\prime}}, \quad\left(x, x^{\prime}\right) \longmapsto x \wedge x^{\prime}
$$

$\left(p, q, p^{\prime}, q^{\prime} \in \mathbf{Z}\right)$, such that the associative law, the existence of an identity element and the anticommutative law

$$
x \wedge x^{\prime}=(-1)^{(p+q)\left(p^{\prime}+q^{\prime}\right)} x^{\prime} \wedge x
$$

hold; (iso)morphisms of bigraded $R$-algebras are defined in an obvious way.

We have constructed an anticommutative bigraded $R$-algebra which we will denote $E(\phi)$. Let

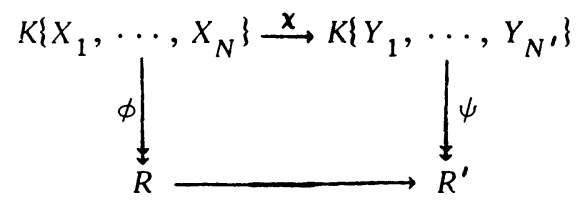


be a commutative diagram of $K$-algebras. Then $\chi^{(\operatorname{ker} \phi)} \subset \operatorname{ker} \psi$, hence $\chi$ induces a morphism of bigraded $R$-algebras $\chi_{*}: E(\phi) \rightarrow E(\psi)$. It is clear that $\chi_{*}$ can be considered as a functor of the diagram (1).

We want to show:

Theorem 1. Let $R$ be an analytic K-algebra. Then $E(\phi)$ is an anticommutative bigraded $R$-algebra, which is uniquely determined up to an isomorphism of bigraded $R$-algebras and may therefore be denoted by $E(R)$.

This means: Let $\psi: \widetilde{R}^{\prime} \rightarrow R$ be another surjective morphism of $K$-algebras, $\tilde{R}^{\prime}=K\left\{Y_{1}, \cdots, Y_{N^{\prime}}\right\}$. Then $E(\psi) \cong E(\phi)$.

Let us first prove this for a special case: Assume $\tilde{R}^{\prime}=K\left\{X_{2}, \cdots, X_{N}\right\} \subset \widetilde{R}$, $\phi\left(X_{1}\right)=0$, and $\phi\left(X_{i}\right)=\psi\left(X_{i}\right)$ for $i=2, \cdots, N$. Let $\iota: \widetilde{R}^{\prime} \rightarrow \widetilde{R}$ be the inclusion map, and $\sigma: \widetilde{R} \rightarrow \widetilde{R}^{\prime}$ be the morphism defined by $\sigma\left(X_{1}\right)=0$ and $\sigma\left(X_{i}\right)=X_{i}$ for $i=2, \cdots, N$. It is enough to prove that $\iota_{*}: E(\psi) \rightarrow E(\phi)$ and $\sigma_{*}: E(\phi) \rightarrow E(\psi)$ are inverses of each other. From $\sigma \iota=$ id we conclude that $\sigma_{*} \iota_{*}=\mathrm{id}$. Now consi$\operatorname{der} \iota_{*} \sigma_{*}=\rho_{*}$, where

$$
\rho:=\iota \sigma: \widetilde{R} \rightarrow \widetilde{R}
$$

$\rho\left(X_{1}\right)=0, \rho\left(X_{i}\right)=X_{i}$ for $i=2, \cdots, N$. From the commutativity of

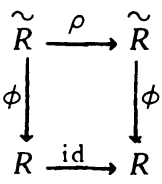

it follows that $\rho(a) \subset a$. Hence the unique extension of $\rho$ to an endomorphism of the differential graded $K$-algebra $\widetilde{\Omega}$ respects the filtration of $\widetilde{\Omega}$, and induces morphisms of chain complexes

$$
\rho: F^{p} \tilde{\Omega} / F^{p+1} \tilde{\Omega} \rightarrow F^{p} \tilde{\Omega} / F^{p+1} \tilde{\Omega}
$$

for all $p \in \mathbf{Z}$. Since we are in characteristic zero, any $\omega \in \widetilde{\Omega}^{n}$ may be written uniquely as

$$
\omega=\omega_{0}+\sum_{k=1}^{\infty}\left(\tilde{d} X_{1}^{k}\right) \wedge \omega_{k}
$$

with $\omega_{0} \in \tilde{R} \Omega^{n}\left(\tilde{R}^{\prime}\right)$ and $\omega_{k} \in \Omega^{n-1}\left(\tilde{R}^{\prime}\right)$ for $k>0$. Let

$$
\zeta(\omega):=\sum_{k=1}^{\infty} X_{1}^{k} \omega_{k} \in \widetilde{\Omega}^{n-1} .
$$

One checks easily that $\zeta$ is a homotopy from the identity of $\tilde{\Omega}$ to $\rho: \tilde{\Omega} \rightarrow \tilde{\Omega}$. Furthermore, 


$$
\zeta\left(F^{p} \widetilde{\Omega}^{n}\right)=\zeta\left(a^{p-n} \widetilde{\Omega}^{n}\right) \subset a^{p+1-n \widetilde{\Omega}^{n-1}}=F^{p} \widetilde{\Omega}^{n-1} .
$$

Hence $\zeta$ induces for each $p \in \mathrm{Z}$ a homotopy

$$
\left(F^{p} \widetilde{\Omega}^{n} / F^{p+1} \widetilde{\Omega}^{n} \rightarrow F^{p} \widetilde{\Omega}^{n-1} / F^{p+1} \widetilde{\Omega}^{n-1}\right)_{n \in Z}
$$

from the identity of $F^{p} \tilde{\Omega} / F^{p+1} \tilde{\Omega}$ to

$$
\rho: F^{p} \tilde{\Omega} / F^{p+1} \tilde{\Omega} \rightarrow F^{p} \tilde{\Omega} / F^{p+1} \tilde{\Omega} .
$$

The existence of such a homotopy proves $\rho_{*}=\mathrm{id}$, and the proof is finished in our special case.

Let us now prove $E(\phi) \cong E(\psi)^{-}$in the general case. Let $u_{i}:=\phi\left(X_{i}\right), v_{j}:=$ $\psi\left(Y_{j}\right)$, and choose $g_{i} \in \psi^{-1}\left(u_{i}\right), b_{j} \in \phi^{-1}\left(v_{j}\right)$ for $i=1, \cdots, N ; j=1, \cdots, N^{\prime}$. Write $X=\left(X_{1}, \cdots, X_{N}\right)$, etc. We have a commutative diagram of $K$-algebras

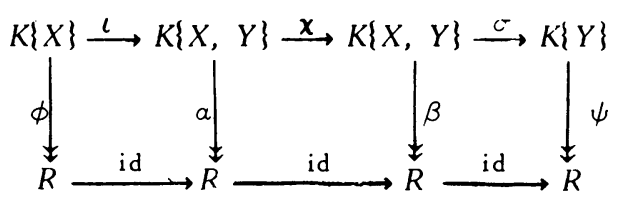

where $\iota, \sigma, \alpha, \beta$ and $\chi$ are defined by

$$
\begin{array}{lll}
\iota(X)=X, & \\
\sigma(X)=0, & \sigma(Y)=Y, \\
\alpha(X)=u, & \alpha(Y)=0, \\
\beta(X)=0, & \beta(Y)=v, \\
\chi(X)=X+g, & \chi(Y)=Y-b(X+g) .
\end{array}
$$

From the special case considered first it follows by induction on $N^{\prime}$ that $\iota_{*}$ is an isomorphism and by induction on $N$ that $\sigma_{*}$ is an isomorphism. Now $\chi$ is an isomorphism since an inverse is given by

$$
\chi^{-1}(X)=X-g(Y+b), \quad \chi^{-1}(Y)=Y+b .
$$

Hence $\chi_{*}$ is an isomorphism. Altogether we have isomorphisms

$$
E(\phi) \stackrel{\iota_{*}}{\longrightarrow} E(\alpha) \stackrel{\boldsymbol{x}_{*}}{\longrightarrow} E(\beta) \stackrel{\sigma_{*}}{\longrightarrow} E(\psi)
$$

and Theorem 1 is completely proved.

3. An $S$-free resolution of $R$. Now let the analytic $K$-algebra $R$ be a complete intersection: There is a surjective morphism $\phi: \widetilde{R} \rightarrow R$, whose kernel $a$ is generated by a prime sequence $\left(a_{1}, \cdots, a_{l}\right)$ in $\tilde{R}=K\left\{X_{1}, \cdots, X_{N}\right\}$. In order to study $\Omega(R)$ we will construct an $S$-free resolution $M$ of $R$, which is a differential graded $S$-algebra at the same time. 
As a graded algebra, let $M$ be the free anticommutative $S$-algebra on generators $f_{1}, \cdots, f_{N}$ of degree 1 and $e_{1}, \cdots, e_{l}$ of degree 2 . This means, for $k \in \mathbf{Z}$, $M_{k}$ is a free $S$-module, and a basis is formed by all formal products $e_{j_{1}} \wedge \cdots \wedge$ $e_{j_{m}} \wedge f_{i_{1}} \wedge \cdots \wedge f_{i_{n}}$, where $1 \leq j_{1} \leq \cdots \leq j_{m} \leq l, 1 \leq i_{1}<\cdots<i_{n} \leq N$, and $k=$ $2 m+n$ (in particular $M_{0}=S$ and $M_{k}=0$ for $k<0$ ). For the multiplication $\wedge$ we have: $e_{j}$ commutes with all elements, while $f_{i} \wedge f_{i}^{\prime}=-f_{i} \wedge f_{i}$.

The augmentation $M_{0} \rightarrow R$ is by definition the augmentation $\pi$ of $S$. The differential $\left(\delta_{k}: M_{k} \rightarrow M_{k-1}\right)_{k \in Z}$ has to be defined on the generators $e_{j}, f_{i}$ only, since it is subject to the Leibniz rule

$$
\delta(x \wedge y)=\delta(x) \wedge y+(-1)^{k} x \wedge \delta(y)
$$

for all $x \in M_{k}, y \in M_{k}$. Before we can define $\delta\left(e_{j}\right)$ and $\delta\left(f_{i}\right)$ we have to introduce a few more notations. For $i=0,1, \cdots, N$ define a morphism of $K$-algebras

$$
\tau_{i}: \widetilde{R} \rightarrow \tilde{S}:=\tilde{R} \Perp \tilde{R}
$$

by

$$
\tau_{i}\left(X_{j}\right):= \begin{cases}X_{j} \otimes 1 & \text { for } 1 \leq j \leq i \\ 1 \otimes X_{j} & \text { for } i<j \leq N\end{cases}
$$

Then

$$
\begin{aligned}
& \Delta:=\tau_{N}-\tau_{0}: \widetilde{R} \rightarrow \tilde{S}, \\
& \partial_{i}:=\frac{1}{\Delta X_{i}}\left(\tau_{i}-\tau_{i-1}\right): \widetilde{R} \rightarrow \tilde{S}
\end{aligned}
$$

$(i=1, \cdots, N)$ are $K$-linear maps. Observe that for all $x \in \widetilde{R}$ we have

$$
\Delta x=\sum_{i=1}^{N} \partial_{i} x \Delta X_{i},
$$

and $\partial_{i} x$ has the image $\partial x / \partial x_{i}$ under the augmentation $\tilde{\pi}: \tilde{S} \rightarrow \tilde{R}$ of $\tilde{S}$. Keeping in mind that every $S$-module is an $\tilde{S}$-module in a natural way, we define

$$
\delta\left(f_{i}\right):=\Delta X_{i} 1 \in M_{0}, \quad \delta\left(e_{j}\right):=\sum_{i=1}^{N} \partial_{i} a_{j} f_{i} \in M_{1} .
$$

Theorem 2. $(M, \delta, \pi)$ is an $S$-free resolution of the complete intersection $R$.

Proof. We have $\delta_{0} \delta_{1}\left(f_{i}\right)=0$ and

$$
\delta_{1} \delta_{2}\left(e_{j}\right)=\sum_{i=1}^{N} \partial_{i} a_{j} \Delta X_{i} 1=\Delta a_{j} 1=0
$$


hence $\delta^{2}=0$. Furthermore,

$$
\pi \delta_{1}\left(f_{i}\right)=\pi\left(\Delta X_{i} 1\right)=X_{i}-X_{i}=0 .
$$

These equations show that $M$ is a complex of free $S$-modules over $R$. We have to show it is acyclic over $R$.

For $l=0, M$ is the Koszul complex defined by the prime sequence $\left(\Delta X_{1}, \cdots\right.$, $\left.\Delta X_{N}\right)$ and the assertion is well known [6, IV, A, \$2]: We obtain an exact sequence

$$
\cdots \rightarrow \widetilde{M}_{2} \stackrel{\tilde{\delta}_{2}}{\longrightarrow} \widetilde{M}_{1} \stackrel{\widetilde{\delta}_{1}}{\longrightarrow} \widetilde{M}_{0} \stackrel{\tilde{\pi}}{\longrightarrow} \widetilde{R} \rightarrow 0
$$

where $\widetilde{M}_{0}=\tilde{S}, \widetilde{M}_{1}$ is a free $\tilde{S}$-module with basis $\left(\tilde{f}_{1}, \cdots, \tilde{f}_{N}\right)$, and $\tilde{M}_{i}=\bigwedge_{\sim}^{\wedge} \underset{\mathcal{M}_{1}}{i}$.

Return to an arbitrary $l \in \mathbf{N}$. An $x \in S$ may be written as a series $\Sigma_{m \in \mathrm{N}} x_{m} \otimes y_{m}$ with $x_{m}, y_{m} \in R$ which converges with respect to the topology defined by the ideal $\left.\left.\sum_{i=1}^{N} X_{i} \otimes 1\right) S+\left(1 \otimes X_{i}\right) S\right)$. Let $x \in$ ker $\pi$. Then $\Sigma x_{m} y_{m}=$ $\pi(x)=0$, hence

$$
\begin{aligned}
x & =\sum_{m \in \mathbf{N}}\left(x_{m} \otimes y_{m}-1 \otimes x_{m} y_{m}\right)=\sum_{m \in \mathbf{N}} \Delta x_{m}\left(1 \otimes y_{m}\right) \\
& =\sum_{i=1}^{N}\left(\sum_{m \in \mathbf{N}} \partial_{i} x_{m}\left(1 \otimes y_{m}\right)\right) \Delta X_{i} \\
& =\delta\left(\sum_{i=1}^{N} \sum_{m \in \mathbf{N}} \partial_{i} x_{m}\left(1 \otimes y_{m}\right) f_{i}\right) .
\end{aligned}
$$

This shows $\operatorname{ker} \pi=\operatorname{im} \delta_{1}$.

An element $x$ of $\operatorname{ker} \delta_{1}$ may be written as $x=\sum_{i=1}^{N} x_{i} f_{i}, x_{i} \in \tilde{S}$, where

$$
\sum_{i=1}^{N} x_{i} \Delta x_{i} \in \operatorname{ker}(\tilde{S} \rightarrow S)=\sum_{j=1}^{l}\left(a_{j} \otimes 1\right) \tilde{S}+\sum_{j=1}^{l} \Delta a \tilde{S} .
$$

Let $u_{j}, v_{j} \in \tilde{S}$ be such that

$$
\begin{aligned}
\sum_{i=1}^{N} x_{i} \Delta X_{i} & =\sum_{j=1}^{l}\left(a_{j} \otimes 1\right) u_{j}+\sum_{j=1}^{l}\left(\Delta a_{j}\right) v_{j} \\
& =\sum_{j=1}^{l}\left(a_{j} \otimes 1\right) u_{j}+\sum_{j=1}^{l} \sum_{i=1}^{N}\left(\partial_{i} a_{j}\right)\left(\Delta X_{i}\right) v_{j}
\end{aligned}
$$

Applying $\tilde{\pi}$ to this equation we obta in

$$
0=\sum_{j=1}^{l} a_{j} \tilde{\pi}\left(u_{j}\right)
$$

It follows that 


$$
\sum_{j=1}^{l}\left(a_{j} \otimes 1\right) u_{j}=\sum_{j=1}^{l}\left(a_{j} \otimes 1\right)\left(u_{j}-\tilde{\pi}\left(u_{j}\right) \otimes 1\right) .
$$

There exist $u_{i j} \in \tilde{S}$ such that

$$
u_{j}-\tilde{\pi}\left(u_{j}\right) \otimes 1=\sum_{i=1}^{N} u_{i j} \Delta X_{i}
$$

hence

$$
0=\sum_{i=1}^{N}\left(x_{i}-\sum_{j=1}^{l}\left(a_{j} \otimes 1\right) u_{i j}-\sum_{j=1}^{l}\left(\partial_{i} a_{j}\right) v_{j}\right) \Delta X_{i}
$$

Consequently,

$$
\sum_{i=1}^{N}\left(x_{i}-\sum_{j=1}^{l}\left(a_{j} \otimes 1\right) u_{i j}-\sum_{j=1}^{l}\left(\partial_{i} a_{j}\right) v_{j}\right) \tilde{f}_{i} \in \operatorname{ker} \widetilde{\delta}_{1}=\operatorname{im} \widetilde{\delta}_{2},
$$

and

$$
\operatorname{im} \delta_{2} \ni \sum_{i=1}^{N}\left(x_{i}-\sum_{j=1}^{l}\left(a_{j} \otimes 1\right) u_{i j}-\sum_{j=1}^{l}\left(\partial_{i} a_{j}\right) v_{j}\right) f_{i}=x-\delta_{2} \sum_{j=1}^{l} v_{j} e_{j}
$$

Hence $x \in \operatorname{im} \delta_{2}$, and we have shown $\operatorname{ker} \delta_{1}=\operatorname{im} \delta_{2}$.

Now we will prove, by induction on $l$, that $M$ is acyclic over $R$. The case $l=0$ being well known (see above) we may assume $l>0$ and that for $R^{\prime}:=$ $\widetilde{R} /\left(a_{1} \tilde{R}+\cdots+a_{l-1} \tilde{R}\right)$ we have an exact sequence

$$
\cdots \rightarrow M_{2}^{\prime} \stackrel{\delta_{2}^{\prime}}{\longrightarrow} M_{1}^{\prime} \stackrel{\delta_{1}^{\prime}}{\longrightarrow} M_{0}^{\prime}=S^{\prime} \stackrel{\pi^{\prime}}{\longrightarrow} R^{\prime} \rightarrow 0
$$

with $M^{\prime}$ a free anticommutative $S^{\prime}$-algebra on generators $f_{1}^{\prime}, \cdots, f_{N}^{\prime}, e_{1}^{\prime}, \cdots$, $e_{l-1}^{\prime}$ as above.

Let $L=\left(L_{i}\right)_{i \in Z}$ be the free anticommutative graded $S$-algebra on one generator $e$ of degree 2; then $L_{i}$ is the free $S$-module with basis $\left(e^{i / 2}\right)$ if $i \in 2 \mathbf{N}$, and $L_{i}=0$ for all other $i$. In particular $L_{0}=S, e^{0}=1$.

We have a unique isomorphism of graded $S$-algebras

$$
\begin{aligned}
& M \longrightarrow M^{\prime} \otimes_{S^{\prime}} L, \\
& f_{i} \longmapsto f_{i}^{\prime} \otimes e^{0}, \\
& e_{j} \longmapsto e_{j}^{\prime} \otimes e^{0} \quad \text { for } j<l, \\
& e_{l} \longmapsto 1 \otimes e .
\end{aligned}
$$

Replacing $M$ by something isomorphic we may assume this is the identity of $M$. Then for all $k \in \mathbf{N}$, 
where

$$
M_{k}=\bigoplus_{m=0}^{[k / 2]} M_{k-2 m}^{\prime} \otimes_{S^{\prime}} e^{m} S=\bigoplus_{m=0}^{[k / 2]} M_{k-2 m}^{\prime \prime} \wedge e^{m}
$$

$$
M^{\prime \prime}:=M^{\prime} \otimes_{S^{\prime}} L_{0} \subset M .
$$

$\operatorname{ker}\left(S^{\prime} \rightarrow S\right)$ is the ideal in $S^{\prime}$ generated by the prime sequence $\left(\left(1 \otimes a_{l}\right) 1\right.$, $\left(\Delta a_{l}\right) 1$ ). Hence the Koszul complex of this prime sequence is acyclic over $S$ (see $[6, \mathrm{IV}, A, \S 2])$ :

$$
\cdots \rightarrow 0 \rightarrow S^{\prime} \stackrel{\eta}{\rightarrow} S^{\prime} \oplus S^{\prime} \stackrel{\theta}{\rightarrow} S^{\prime} \rightarrow 0
$$

where

$$
\begin{aligned}
\eta(x): & =\left(-\Delta a_{l}, a_{l} \otimes 1\right) x \\
\theta(x, y): & =\left(a_{l} \otimes 1\right) x+\left(\Delta a_{l}\right) y
\end{aligned}
$$

for all $x, y \in S^{\prime}$. Applying $R^{\prime} \otimes_{S^{\prime}}$ we get

$$
\begin{aligned}
\cdots \rightarrow 0 \rightarrow R^{\prime} & \longrightarrow R^{\prime} \oplus R^{\prime} \longrightarrow R^{\prime} \rightarrow 0 \\
x & \longmapsto\left(0, a_{l} x\right) \\
(x, y) & \longmapsto a_{l} x .
\end{aligned}
$$

Here $R^{\prime} \rightarrow R^{\prime} \oplus R^{\prime}$ is injective, because $a_{l} 1$ is not a zero-divisor in $R^{\prime}$. The homology groups of this complex are

$$
\operatorname{Tor}_{k}^{S^{\prime}}\left(R^{\prime}, S\right) \begin{cases}\cong R & \text { for } k=0,1 \\ =0 & \text { otherwise. }\end{cases}
$$

We use only the latter result. It shows that

$$
\cdots \rightarrow M_{3}^{\prime \prime} \rightarrow M_{2}^{\prime \prime} \rightarrow M_{1}^{\prime \prime}
$$

is exact.

Now we will show that for $k>1$ any given element of $H_{k}(M)$ is zero. Take the smallest $m \in\{-1\} \cup \mathbf{N}$, such that the given element is represented by a cycle

$$
x \in \bigoplus_{i=0}^{m} M_{k-2 i}^{\prime \prime} \wedge e^{i} .
$$

$m=-1$ is equivalent to $x=0$. Therefore, let us derive a contradiction from the assumption $m \geq 0$. Let $y \wedge e^{m}$ be the component of $x$ in $M_{k-2 m}^{\prime \prime} \wedge e^{m}$, where $y \in M_{k-2 m}^{\prime \prime}$. We distinguish three cases.

Case 1. $k-2 m>1$. From $\delta(x)=0$ it follows $\delta(y)=0$. This implies $y=$ $\delta(z)$ for some $z \in M_{k-2 m+1}^{\prime \prime}$. 


$$
y \wedge e^{m}=\delta\left(z \wedge e^{m}\right)-m \delta(e) \wedge z \wedge e^{m-1} .
$$

Case 2. $k-2 m=1$. From $\delta(x)=0$ it follows $\delta(y)=0$. This implies $y=$ $\delta\left(z+z_{1} e\right)$ for some $z \in M_{2}^{\prime \prime}$ and $z_{1} \in S$.

$$
y \wedge e^{m}=\delta\left(z \wedge e^{m}+\left(z_{1} /(m+1)\right) e^{m+1}\right)-m \delta(e) \wedge z / \cdot e^{m-1} .
$$

Case 3. $k-2 m=0$. Let $y_{1} \wedge e^{m-1}$ be the component of $x$ in $M_{2}^{\prime \prime} \wedge e^{m-1}$, where $y_{1} \in M_{2}^{\prime \prime}$. From $\delta(x)=0$ it follows

$$
0=m y \delta(e)+\delta\left(y_{1}\right)=\delta\left(m y e+y_{1}\right) .
$$

This implies, as we will show subsequently, my $\epsilon$ im $\delta_{1}$. Hence there is a $z \in M_{1}$ such that $y=\delta(z)$, and (2) holds as in Case 1 .

In all three cases

$$
y \wedge e^{m} \equiv-m \delta(e) \wedge z \wedge e^{m-1} \bmod \operatorname{im} \delta_{k+1},
$$

a contradiction to the minimal choice of $m$.

We still have to prove: If, for some $y \in S$, ye $\in M_{2}^{\prime \prime}+\operatorname{ker} \delta_{2}$, then $y \in \operatorname{im} \delta_{1}$. Consider the exact and commutative diagram

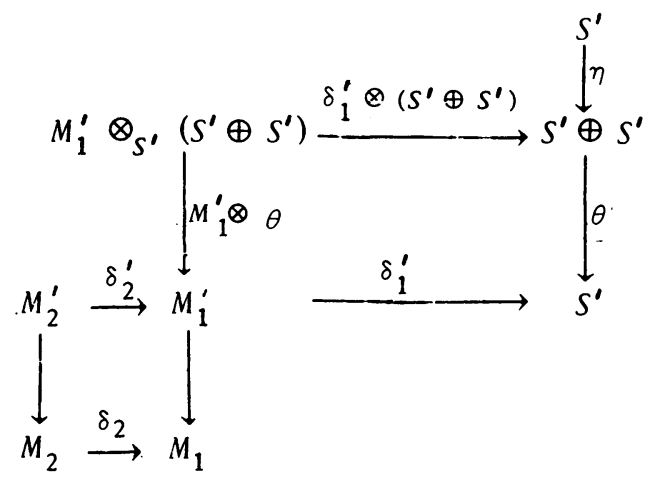

where $M_{i}^{\prime} \rightarrow M_{i}$ are canonical morphisms. Let $y^{\prime} \in S^{\prime}$ be a counterimage of $y$. From

$$
\delta_{2}(y e)=y \sum_{i=1}^{N} \partial_{i} a_{l} f_{i} \in \operatorname{im}\left(M_{2}^{\prime} \rightarrow M_{1}\right)
$$

we see

$$
\begin{aligned}
y^{\prime} \sum_{i=1}^{N} \partial_{i} a_{l} f_{i}^{\prime} & \epsilon \operatorname{im} \delta_{2}^{\prime}+\operatorname{ker}\left(M_{1}^{\prime} \rightarrow M_{1}\right) \\
& =\operatorname{ker} \delta_{1}^{\prime}+\operatorname{im}\left(M_{1}^{\prime} \otimes \theta\right)
\end{aligned}
$$

This shows 


$$
\begin{aligned}
\theta\left(0, y^{\prime}\right) & =\Delta a_{l} y^{\prime}=\delta_{1}^{\prime}\left(y^{\prime} \sum_{i=1}^{N} \partial_{i} a_{l} f_{i}^{\prime}\right) \\
& \in \operatorname{im} \theta\left(\delta_{1}^{\prime} \otimes\left(S^{\prime} \oplus S^{\prime}\right)\right),
\end{aligned}
$$

hence $\left(0, y^{\prime}\right) \in \operatorname{im} \eta+\left(\operatorname{im} \delta_{1}^{\prime} \oplus \operatorname{im} \delta_{1}^{\prime}\right)$, and $y^{\prime} \in\left(a_{l} \otimes 1\right) S^{\prime}+$ im $\delta_{1}^{\prime}$. This implies $y \in \operatorname{im} \delta_{1}$. Q.E.D.

The proof of Theorem 2 is complete.

Remark. Let $R^{*}$ be any analytic $K$-algebra, $R=R^{*} / i$ where $i$ is an ideal generated by a prime sequence. Let $S^{*}$ be the enveloping algebra of $R^{*}$ and $\pi^{*}$ its augmentation. Suppose there is given a differential graded $S^{*}$-algebra which is an $S^{*}$-free resolution of $R^{*}$ with the augmentation $\pi^{*}$. Then by the above procedure one can construct a differential graded $S$-algebra which is an $S$-free resolution of $R$ with the augmentation $\pi$.

4. The isomorphy of $E(R)$ and $\Omega(R)$. Let $R$ be a fixed analytic $K$-algebra which is a complete intersection. We will write $\Omega$ instead of $\Omega(R)$, and $E$ instead of $E(R)$. For $m \in \mathbf{Z}$ let

$$
E^{m}:=\bigoplus_{q \in Z} E^{m+q, q}
$$

The multiplication on $E$ makes $\left(E^{m}\right)_{m \in Z}$ an anticommutative graded $R$-algebra. Let us, from now on, denote this graded algebra by $E$.

Theorem 3. $\Omega$ and $E$ are isomorphic graded $R$-algebras.

Proof. Let $B:=\sum_{j=1}^{l} e_{j} S \subset M_{2}$ and let $\mathrm{S}$ denote the symmetric algebra. We may identify the graded $S$-algebra $M$ in a canonical way with $S_{S} B \otimes_{S} \bigwedge_{S} M_{1}$. Let $\bar{B}:=B \otimes_{S} R$ and $\bar{M}:=M \otimes_{S} R$. Let $\bar{f}_{i}, \bar{e}_{j} \in \bar{M}$ be the images of $f_{i}$, $e_{j}$ under the canonical morphism

$$
M \cong M \otimes_{S} S \stackrel{\operatorname{id} \otimes \pi}{\longrightarrow} \bar{M},
$$

and $\bar{\delta}:=\delta \otimes \mathrm{id}_{R} \cdot \bar{M}_{1}$ and $\bar{B}$ are free $R$-modules with bases $\left(\bar{f}_{1}, \cdots, \bar{f}_{N}\right)$ and $\left(\bar{e}_{1}, \cdots, \bar{e}_{l}\right)$ respectively. We may identify the graded $R$-algebra $\bar{M}$ with $\mathrm{S}_{R} \bar{B} \otimes_{R} \bigwedge_{R} \bar{M}_{1}$ in a canonical way. We have

$$
\begin{aligned}
\bar{\delta}\left(\bar{f}_{i}\right) & =0 \quad \text { for } i=1, \ldots, N ; \\
\bar{\delta}\left(\bar{e}_{j}\right) & =\sum_{i=1}^{N} \frac{\partial a_{j}}{\partial X_{i}} \bar{f}_{i} \text { for } j=1, \ldots, l ; \\
\Omega & =H(\bar{M}) ;
\end{aligned}
$$

and an isomorphism of $R$-modules 


$$
\widetilde{\Omega}^{1} \otimes_{\widetilde{R}} R \cong \bar{M}_{1},
$$

where $\bar{f}_{i}$ corresponds to $\tilde{\sim} X_{i} \otimes 1$.

Let $A$ be a free $\tilde{R}$-module with basis $\left(\tilde{e}_{1}, \ldots, \tilde{e}_{l}\right)$. Since $\left(a_{1}, \cdots, a_{l}\right)$ is a prime sequence in $\widetilde{R}$, we have an exact sequence of $\widetilde{R}$-modules

$$
\bigwedge_{\tilde{R}}^{2} A \stackrel{\mathrm{x}_{2}}{\longrightarrow} A \stackrel{\mathrm{x}_{1}}{\longrightarrow} \widetilde{R} \stackrel{\phi}{\longrightarrow} R \rightarrow 0,
$$

where $\chi_{1}\left(\tilde{e}_{j}\right)=a_{j}, \chi_{2}\left(\tilde{e}_{j} \wedge \tilde{e}_{j}{ }^{\prime}\right)=a_{j} \tilde{e}_{j}{ }^{\prime}-a_{j}{ }^{\prime} \tilde{e}_{j}$. There exist canonical isomorphisms

$$
a \otimes_{\widetilde{R}} R \cong A /\left(\operatorname{im} \chi_{2}+a A\right)=A / a A \cong A \otimes_{\widetilde{R}} R \cong \bar{B}
$$

where in the last isomorphism $\bar{e}_{j}$ corresponds to $\tilde{e}_{j} \otimes 1$.

Because $a$ is generated by a prime sequence, the canonical morphisms $\mathrm{S}_{\widetilde{R}}^{m} a \rightarrow a^{m}$ of $\widetilde{R}$-modules $(m \in \mathbf{N}$ ) are isomorphisms which altogether give an isomorphism

$$
\mathrm{S}_{\widetilde{R}} a \rightarrow \bigoplus_{m \in \mathrm{N}} a^{m}
$$

of graded $\widetilde{R}$-modules from the symmetric algebra of a to the Rees algebra of a (cf. [4]).

Now we claim for $n, p \in \mathbf{Z}$,

$$
F^{p} \widetilde{\Omega}^{n} / F^{p+1} \widetilde{\Omega}^{n} \cong\left(\begin{array}{c}
p-n \\
\mathrm{~S}_{R} \bar{B}
\end{array}\right) \otimes_{R} \bigwedge_{R}^{n} \bar{M}_{1} .
$$

For $n>p$ this is trivial since both sides vanish. Let $n \leq p$. Then

$$
\begin{aligned}
& F^{p} \widetilde{\Omega}^{n} / F^{p+1} \widetilde{\Omega}^{n}=a^{p-n} \widetilde{\Omega}^{n} / a^{p-n+1} \widetilde{\Omega}^{n} \\
& \cong\left(a^{p-n} \otimes_{\widetilde{R}} R\right) \otimes_{R}\left(\widetilde{\Omega}^{n} \otimes_{\widetilde{R}} R\right) \\
& \cong\left(\left(\begin{array}{c}
p-n \\
\mathrm{~S}_{\widetilde{R}} a
\end{array}\right) \otimes_{\widetilde{R}} R\right) \otimes_{R}\left(\left(\bigwedge_{R^{n}}^{n} \widetilde{\Omega}^{1}\right) \otimes_{\widetilde{R}} R\right) \\
& \cong\left(\sum_{R}^{p-n}\left(a \otimes_{\widetilde{R}} R\right)\right) \otimes_{R} \bigwedge_{R}^{n}\left(\widetilde{\Omega}^{1} \otimes_{\widetilde{R}} R\right) \\
& \cong\left({ }^{p-n} \bar{S}_{R}^{B}\right) \otimes_{R} \bigwedge_{R}^{n} \bar{M}_{1}
\end{aligned}
$$

as asserted. 
We introduce a grading on the algebra $G \tilde{\Omega}$ :

$$
(G \widetilde{\Omega})_{m}:=\bigoplus_{q \in Z} F^{m+q} \widetilde{\Omega}^{m+2 q} / F^{m+q+1} \widetilde{\Omega}^{m+2 q} .
$$

The differential on $G \tilde{\Omega}$ is of degree - 1, i.e. we have a complex

$$
\cdots \rightarrow(G \widetilde{\Omega})_{2} \rightarrow(G \widetilde{\Omega})_{1} \rightarrow(G \widetilde{\Omega})_{0} \rightarrow \cdots,
$$

and $E=H(G \widetilde{\Omega})$.

From (3) we obtain isomorphisms

$$
(G \widetilde{\Omega})_{m} \cong \bigoplus_{q \in Z}\left(\begin{array}{c}
-q \\
\mathrm{~S}_{R} \bar{B}
\end{array}\right) \otimes_{R} \stackrel{m+2 q}{\bigwedge_{R}} \bar{M}_{1}=\bar{M}_{m}
$$

of $R$-modules for all $m \in \mathbf{Z}$, which yield an isomorphism $G \tilde{\Omega}=\bar{M}$ of differential graded algebras. We conclude

$$
E=H(G \widetilde{\Omega}) \cong H(\bar{M})=\Omega . \quad \text { Q.E.D. }
$$

5. The analytic $K$-al gebra of a hypersurface. By the analytic $K$-algebra of a hypersurface we mean a $K$-algebra isomorphic to $\tilde{R} / a$ where $a$ is a principal ideal in $\widetilde{R}=K\left\{X_{1}, \cdots, X_{N}\right\}$. Since $\widetilde{R}$ is an integral domain the analytic $K$-algebras of hypersurfaces are the simplest complete intersections, and Theorems 1, 2 and 3 apply.

Let $R$ be an analytic $K$-algebra. By $\sqrt{0}$ we denote the nilradical of $R$. $R$ is called reduced, if $\sqrt{0}=\{0\}$. We call reduction of $\Omega(R)$ the morphism

$$
\text { red: } \Omega(R) \rightarrow \Omega(R / \sqrt{0})
$$

induced by the canonical morphism $R \rightarrow R / \sqrt{0}$. If $C$ is an $R$-module, and $D$ the set of non-zero-divisors of $R$, we call the kernel of the canonical morphism $C \rightarrow C_{D}$ torsion submodule TC of $C$ and its elements the torsion elements of $C$.

Proposition. Let $R$ be the analytic K-algebra of a bypersurface, $p, q \in \mathbf{Z}$, $q<0$. Then the R-module $E^{p, q}(R)$ is isomorphic to the $R$-submodule

$$
\operatorname{red}^{-1}\left(\mathrm{~T}^{p+q} \Lambda_{R / \sqrt{0}}^{1}(R / \sqrt{0})\right)
$$

of $\bigwedge_{R}^{p+q} \Omega^{1}(R)$. In particular, if $R$ is reduced,

$$
E^{p, q}(R) \cong \mathrm{T} \bigwedge_{R}^{p+q} \Omega^{1}(R)
$$

Proof. We use notations as in the preceding sections. Let $a=a \tilde{R}$; we only need to consider the case $a \neq 0$. For $x \in \tilde{R}$ let $\operatorname{grad} x:=\Sigma_{i=1}^{N}\left(\partial x / \partial X_{i}\right) \bar{f}_{i} \in \bar{M}_{1}$; $n:=p+q$. 


$$
\begin{aligned}
& E^{p, q}=H\left(F^{p} \tilde{\Omega}^{n-1} \rightarrow F^{p} \widetilde{\Omega}^{n} / F^{p+1} \widetilde{\Omega}^{n} \rightarrow \widetilde{\Omega}^{n+1} / F^{p+1} \widetilde{\Omega}^{n+1}\right) \\
& =H\left(a^{1-q} \widetilde{\Omega}^{n-1} \rightarrow a^{-q} \widetilde{\Omega}^{n} / a^{1-q} \widetilde{\Omega}^{n} \rightarrow \tilde{\Omega} / a^{-q} \widetilde{\Omega}\right) \\
& \cong\left\{x \in a^{-q} \widetilde{\Omega}^{n}: \widetilde{d} x \in a^{-q} \widetilde{\Omega}\right\} /\left(a^{1-q} \widetilde{\Omega}^{n}+\widetilde{d}_{1} 1 \cdots q \wedge \widetilde{\Omega}^{n-1}\right) \\
& =\left\{a^{-q} y: y \in \widetilde{\Omega}^{n}, y \wedge \tilde{d} a \in a \widetilde{\Omega}\right\} /\left(a^{1-c} \widetilde{\Omega}^{n}+a^{-q} \widetilde{d} a \wedge \widetilde{\Omega}^{n-1}\right) \\
& \cong\left\{y \in \widetilde{\Omega}^{n}: y \wedge \widetilde{d} a \in a \widetilde{\Omega}\right\} /\left(a \widetilde{\Omega}^{n}+\widetilde{d} a \wedge \widetilde{\Omega}^{n-1}\right) \\
& \cong\left\{z \in \bigwedge_{R}^{n} \Omega^{1}: u \wedge \operatorname{grad} a=0\right. \\
& \text { for some representative } \left.u \in \bigwedge_{R}^{n} \bar{M}_{1} \text { of } z\right\} \text {. }
\end{aligned}
$$

Now our assertion follows immediately from the following:

Lemma. $u \in \bigwedge_{R}^{n} \bar{M}_{1}$ represents a torsion element of the $(R / \sqrt{0})$-module

$$
\bigwedge_{R / \sqrt{0}}^{n} \Omega^{1}(R / \sqrt{0}) \cong\left\{\begin{array}{l}
\left(\bigwedge_{R}^{n} \Omega^{1}\right) /\left(d \sqrt{0} \wedge \bigwedge_{R}^{n-1} \Omega^{1}\right) \text { for } n \neq 0, \\
R / \sqrt{0} \text { for } n=0,
\end{array}\right.
$$

if and only if $u \wedge \operatorname{grad} a=0$.

Proof of the lemma. Let $p_{1}, \cdots, p_{t}$ be the (pairwise relatively prime) irreducible factors of $a$ in $\widetilde{R}, a=\Pi_{s=1}^{t} p_{s}^{k_{s}}$. Let $b:=\Pi_{s=1}^{t} p_{s} \in \widetilde{R}$. Then $\sqrt{0}=b R$, and $\operatorname{grad} a \wedge \operatorname{grad} b=0$.

"Only if." Suppose there exists an $x \in R$, such that $x u \in \operatorname{grad} a \cdot \wedge \wedge_{R}^{n-1} \bar{M}_{1}$ $+\operatorname{grad} b \wedge \bigwedge_{R}^{n-1} \bar{M}_{1}+b \bigwedge_{R}^{n} \bar{M}_{1}$, and $x$ does not represent a zero-divisor in $R / \sqrt{0}$. Then $x u \wedge \operatorname{grad} a=0$, and $x$ has a representative in $\tilde{R}$ which has no common divisor with $b$, hence with $a$, i.e. $x$ is not a zero-divisor in $R$. It follows that $u \wedge \operatorname{grad} a=0$.

"If." Consider the special case, where $a$ is irreducible. Then $R$ is integral, $R_{D}$ a field. From $u \wedge \operatorname{grad} a=0$ it follows $u=\operatorname{grad} a \wedge y$ for some $y \epsilon$ $\left(\bigwedge_{R}^{n-1} \bar{M}_{1}\right)_{D}$. Let $x \in D$ be such that $x y \in \bigwedge_{R}^{n-1} \bar{M}_{1}$. Then $x u=\operatorname{grad} a \wedge x y$ represents $0 \in \bigwedge_{R}^{n} \Omega^{1}$, and $u$ represents a torsion element of $\bigwedge_{R}^{n} \Omega^{1}$.

Now let $a$ be arbitrary. Let $\tilde{u}$ be a counterimage of $u$ under $\widetilde{\Omega} \rightarrow \bigwedge_{R} \bar{M}_{1}$. For all $s=1, \cdots, t$ we have

$$
\begin{aligned}
\widetilde{d} a & \equiv k_{s}\left(a / p_{s}\right) \widetilde{d} p_{s} \bmod p_{s}^{k_{s}} \tilde{\Omega}, \\
0 & \equiv \widetilde{u} \wedge \tilde{d} a \equiv \tilde{u} \wedge k_{s}\left(a / p_{s}\right) \tilde{d} p_{s} \bmod p_{s}^{k} \widetilde{\Omega} \\
0 & \equiv \tilde{u} \wedge \tilde{d} p_{s} \bmod p_{s} \tilde{\Omega} .
\end{aligned}
$$


According to the special case treated first, $u$ represents a torsion element of the $\left(R / p_{s} R\right)$-module

$$
\left(\bigwedge_{R}^{n} \bar{M}_{1}\right) /\left(\operatorname{grad} p_{s} \wedge \Lambda^{n-1} \bigwedge_{R} \bar{M}_{1}+p_{s} \bigwedge_{R}^{n} \bar{M}_{1}\right)
$$

Hence there exist $x_{s} \in R \backslash p_{s} R$ and $y_{s} \in \bigwedge_{R}^{n-1} \bar{M}_{1}$ such that

$$
x_{s} u \equiv y_{s} \wedge \operatorname{grad} p_{s} \bmod p_{s} \bar{M} \text {. }
$$

Let

$$
x:=\sum_{s=1}^{t}\left(\frac{b}{p_{s}}\right)^{2} x_{s}, \quad y:=\sum_{s=1}^{t} \frac{b}{p_{s}} y_{s} .
$$

Then again for all $s$ we have $\left(\bmod p_{s} \bar{M}\right): x \not \equiv 0$, and

$$
x u \equiv\left(\frac{b}{p_{s}}\right)^{2} x_{s} u \equiv\left(\frac{b}{p_{s}} y_{s}\right) \wedge\left(\frac{b}{p_{s}} \operatorname{grad} p_{s}\right) \equiv y \wedge \operatorname{grad} b .
$$

Therefore, $x$ represents a non-zero-divisor of $R / \sqrt{0}$,

$$
x u \in \operatorname{grad} b \wedge \bigwedge_{R}^{n-1} \bar{M}_{1}+b \bar{M}
$$

and the assertion follows.

Corollary 1. For the analytic algebra $R$ of a bypersurface we bave

(a) $E^{p,-p}(R) \cong \sqrt{0}$ for $0<p \in \mathbf{N}$;

(b) $\Omega^{2}(R) \cong \bigwedge_{R}^{2} \Omega^{1}(R) \oplus \sqrt{0}$;

(c) $R$ is reduced if and only if $\Omega^{2}(R)=\bigwedge_{R}^{2} \Omega^{1}(R)$;

(d) $R$ is normal and reduced if and only if $\Omega^{i}(R)=\bigwedge_{R}^{i} \Omega^{1}(R)$ for $i \leq 3$.

Proof. (a) follows from the Proposition, (b) from (a), and (c) from (b). According to (c), $R$ is reduced if and only if $\Omega^{i}(R)=\bigwedge_{R}^{i} \Omega^{1}(R)$ for $i<3$. Assume $R$ reduced. Then it is well known (see e.g. $[2, \$ 3.5]$ ) that $R$ is normal if and only if $T \Omega^{1}(R)=0$, and (d) follows.

For a reduced complete intersection $R$ the modules $T\left(\bigwedge_{R}^{n} \Omega^{1}(R)\right)$ are of some interest. Vetter [7] proved for the local case $\mathrm{T}\left(\bigwedge_{R}^{i} \Omega^{1}(R)\right)=0$ for all $i \leq n$ if and only if the singular locus of $R$ has codimension $>n$ (the empty set has codimension $\infty$ ). From this we get

Corollary 2. For the local analytic algebra $R$ of a bypersurface we bave $\Omega^{i}(R)=\bigwedge_{R}^{i} \Omega^{1}(R)$ for all $i \leq r+1$ if and only if the singular locus of $R$ bas codimension $\geq r$. 


\section{REFERENCES}

1. H. Cartan and S. Eilenberg, Homological algebra, Princeton Univ. Press, Princeton ton, N. J., 1956. MR 17, 1040.

2. R. Berger, R. Kiehl, E. Kunz and H.-J. Nastold, Differentialrechnung in der analytischen Geometrie, Lecture Notes in Math., no. 38, Springer-Verlag, Berlin and New York, 1967. MR 37 \#469.

3. G. Hochschild, B. Kostant and A. Rosenberg, Differential forms on regular affine algebras, Trans. Amer. Math. Soc. 102 (1962), 383-408. MR 26 \#167.

4. A. Micali, Sur ses algèbres universelles, Ann. Inst. Fourier (Grenoble) 14 (1964), fasc. 2, 33-87. MR 31 \#1275.

5. G. S. Rinehart, Differential forms on general commutative algebras, Trans. Amer. Math. Soc. 108 (1963), 195-222. MR 27 \#4850.

6. J.-P. Serre, Algèbre locale. Multiplicités, Cours au Collège de France, 1957/58, Lecture Notes in Math., no. 11, Springer-Verlag, Berlin and New York, 1965. MR 34 $\# 1352$.

7. U. Vetter, Äussere Potenzen von Differentialmoduln reduzierter vollständiger Durchschnitte, Manuscripta Math. 2(1970), 67-75. MR 42 \#255.

8. K. Wolffhardt, Zur Homologietheorie der assoziativen Algebren, Manuscripta Math. $4(1971), 149-168$.

MATHEMATISCHES INSTITUT, UNIVERSITÄT MÜNCHEN, MÜNCHEN, FEDERAL REPUBLIC OF GERMANY 